\title{
An Overview of Hybrid Possibilistic Reasoning
}

\author{
Churn-Jung Liau \\ Institute of Information Science \\ Academia Sinica, Taipei, Taiwan \\ liaucj@iis.sinica.edu.tw
}

\begin{abstract}
The objective of this paper is to introduce the hybrid logic methodology into possibilistic reasoning. It has been well-known that possibilistic logic has some strong modal logic flavor. However, modal logic lacks the capability of referring to states or possible worlds though states are crucial to its semantics. Hybrid logic circumvents the problem by blending the classical logic mechanism into modal logic. It is a hybrid of classical logic and modal logic, however, unlike classical logic, it treats terms and formulas uniformly. We study some variants of hybrid possibilistic logic, including hybrid qualitative possibility logic, hybrid graded modal logic, and hybrid possibilistic description logic. The syntax and semantics of the logics are presented. Some possible applications of the proposed logics are also suggested.
\end{abstract}

Key words: Possibilistic logic, Qualitative possibility logic, Hybrid logic, Graded modal logic, Description Logic.

\section{Introduction}

Knowledge representation and reasoning is fundamental to knowledge based systems. In practice, the acquired knowledge is rarely certain. To represent and reason about uncertain knowledge, many uncertainty reasoning methods have been proposed and extensively studied. Among them, possibilistic logic[4] is a logic of partial ignorance and/or inconsistency based on possibility theory[11]. It has been shown that possibilistic reasoning can be formulated in a kind of graded modal logic $[5,7]$. This kind of graded modal logic can do not only possibilistic but also similarity-based reasoning[5], so it substantially extends the application scope of possibilistic logic.

The standard modal logic(ML) is based on the so-called Kripke semantics. The basic entities of a Kripke model are possible worlds (or states) and the binary relations over them. However, in modal logic, we can only talk about the unary relations by propositional wffs and the binary ones by modal wffs. What is lacking in modal logic is thus the capability of referring to the individuals or states explicitly. This means that modal logic is sometimes an inadequate representation formalism for applications.

The hybrid logic paradigm is an attempt to correct the situation[1]. It is called hybrid logic because it can be seen as a hybrid of classical and modal logic. The key idea of hybrid logic is the treatment of terms (or individuals, states, etc.) as formulas. 
The purpose of this paper is to introduce the hybrid methodology into possibilistic reasoning. Though it is a followup of [6], we will focus on the general methodology instead of the further technical development. In the following, we will first review the hybrid movement in the ordinary modal logic and then show how the development can be mimicked in the realm of possibilistic reasoning.

\section{Review: The Hybrid Logic Methodology}

\subsection{Basic hybrid logic}

One of the most basic HL is to add nominals and satisfaction operator to the ML[1]. The wffs of HL is defined by

$$
W F F:=p|a| \neg \varphi|\varphi \wedge \psi| \varphi \vee \psi|\varphi \supset \psi|[\alpha] \varphi|\langle\alpha\rangle \varphi| a: \varphi .
$$

For the semantics, a Kripke frame is a pair $\left(W,\left(R_{i}\right)_{i \geq 0}\right)$ where $W$ is a set of possible worlds and each $R_{i}$ is a binary relation over $W$ and an HL model $\mathcal{M}$ is a triple $\left(W,\left(R_{i}\right)_{i \geq 0}, V\right)$, where $\left(W,\left(R_{i}\right)_{i \geq 0}\right)$ is the underlying Kripke frame and $V$ is a truth assignment which assigns to each propositional symbol a subset of $W$ and each nominal a singleton subset of $W$. By somewhat abusing the notation, we will also write $V(a)$ for the possible world $w$ if it is the unique element of $V(a)$. The satisfaction of a wff with respect to an HL model $\mathcal{M}$ and a possible world $w$ is defined as follows:

1. the classical wffs are defined as usual.

2. $\mathcal{M}, w=\left[\alpha_{i}\right] \varphi$ iff for all $\left(w, w^{\prime}\right) \in R_{i}, \mathcal{M}, w^{\prime} \models \varphi$

3. $\mathcal{M}, w \mid=\left\langle\alpha_{i}\right\rangle \varphi$ iff there exists $\left(w, w^{\prime}\right) \in R_{i}$ such that $\mathcal{M}, w^{\prime} \models \varphi$

4. $\mathcal{M}, w=a$ iff $V(a)=\{w\}$

5. $\mathcal{M}, w \mid=a: \varphi$ iff $\mathcal{M}, V(a) \models \varphi$

According to the semantics, the nominals are interpreted as names of the possible states. Thus the near-atommic satisfaction statement $a: b$ asserts that the states named by $a$ and $b$ are identical. This makes it possible to reason about the equality of states. Similarly $a:\left\langle\alpha_{i}\right\rangle b$ means that the state named by $b$ is an $R_{i}$-successor of the state named by $a$, so HL has the capability of making assertions about the relations that hold between specific states. In summary, HL brings to ML the classical concepts of identity and reference.

\subsection{Description logic}

The idea of extending modal logics with the capability of reasoning about specific individuals is not new to the knowledge representation community. The idea is fully developed under the title of description logic (DL). Nowadays, there have been many variants of DL's. Here, we introduce one of them, called $\mathcal{A L C}[10]$. Though $\mathcal{A L C}$ is a rather restricted sub-language of many description logics, it possesses the most essential features of these logics. 
The alphabets of $\mathcal{A L C}$ consists of three disjoint sets, the elements of which are called concept names, role names, and individual names respectively. The roles terms of $\mathcal{A L C}$ are just role names and denoted by $R$ (sometimes with subscripts) and the concept terms are formed according to the following rules.

$$
C::=A|\top| \perp|C \sqcap D| C \sqcup D|\neg C| \forall R: C \mid \exists R: C
$$

where $A$ is metavariable for concept names, $R$ for role terms and $C$ and $D$ for concept terms. The wffs of $\mathcal{A L C}$ consists of terminological and assertional formulas. Their formation rules are as follows.

1. If $C$ and $D$ are concept terms, then $C=D$ is a terminological formula.

2. If $C$ is a concept term, $R$ is a role term, and $a, b$ are individual names, then $R(a, b)$ and $C(a)$ are assertional formulas.

The terminological formula $C \sqcap \neg D=\perp$ is abbreviated as $C \sqsubseteq D$.

The Tarskian semantics for $\mathcal{A L C}$ are given by assigning sets to concept names and binary relations to roles names. Formally, an interpretation for $\mathcal{A L C}$ is a pair $\mathcal{I}=(U, \rrbracket \cdot \rrbracket)$, where $U$ is a set of universe and $\rrbracket \cdot \rrbracket$ is an interpretation function which assigns to each concept name a subset of $U$, each role name a subset of $U \times U$, and each individual name an element of $U$. The domain of $\rrbracket \cdot \rrbracket$ can be extended to all concept terms by induction

1. $\llbracket \top \rrbracket=U$ and $\llbracket \perp \rrbracket=\emptyset$.

2. $\rrbracket \neg C \rrbracket=U \backslash \rrbracket C \rrbracket$, $\llbracket C \sqcap D \rrbracket=\rrbracket C \rrbracket \cap \rrbracket D \rrbracket$, and $\llbracket C \sqcup D \rrbracket=\rrbracket C \rrbracket \cup \rrbracket D \rrbracket$.

3. $\llbracket \forall R: C \rrbracket=\{x \mid \forall y((x, y) \in \llbracket R \rrbracket \Rightarrow y \in \llbracket C \rrbracket)\}$

4. $\llbracket \exists R: C \rrbracket=\{x \mid \exists y((x, y) \in \llbracket R \rrbracket \wedge y \in \llbracket C \rrbracket)\}$

An interpretation $\mathcal{I}=(U, \| \cdot \rrbracket)$ satisfies a wff

$$
\begin{gathered}
C=D \Leftrightarrow \llbracket C \rrbracket=\rrbracket D \rrbracket, \\
R(a, b) \Leftrightarrow(\llbracket a \rrbracket, \llbracket b \rrbracket) \in \llbracket R \rrbracket, \\
C(a) \Leftrightarrow \llbracket a \rrbracket \in \llbracket C \rrbracket .
\end{gathered}
$$

If $\mathcal{I}$ satisfies a wff $\varphi$, it will be written as $\mathcal{I} \models \varphi$. A set of wffs $\Sigma$ is said to be satisfied by $\mathcal{I}$, written as $\mathcal{I}=\Sigma$, if $\mathcal{I}$ satisfies each wff of $\Sigma$ and $\Sigma$ is satisfiable if it is satisfied by some $\mathcal{I}$. A wff $\varphi$ is an $\mathcal{A L C}$-consequence of $\Sigma$, denoted by $\Sigma \models{ }_{\mathcal{A L C}} \varphi$ or simply $\Sigma \models \varphi$, iff for all interpretations $\mathcal{I}, \mathcal{I} \models \Sigma$ implies $\mathcal{I} \models \varphi$, and $\varphi$ is $\mathcal{A L C}$-valid if it is the $\mathcal{A L C}$-consequence of $\emptyset$.

Without the assertional formulas, a DL is nothing more than an ML if we consider each concept term in DL as a wff in ML. However, the assertional wffs $C(a)$ and $R(a, b)$ corresponds exactly to the satisfaction statements $a: \varphi$ and $a:\langle\alpha\rangle b$ in HL. Thus DL also provide a similar expressive power as HL. However, there is some subtle difference between DL and HL. While nominals in HL are treated as wffs, individual names are not treated as concepts in DL. In HL, every thing is treated equally. They are all wffs. 


\section{Review: Possibilistic Logic}

Possibility theory is developed by Zadeh from fuzzy set theory[11]. Given a universe $W$, a possibility distribution on $W$ is a function $\pi: W \rightarrow[0,1]$. In general, the normalized condition is required, i.e., $\sup _{w \in W} \pi(w)=1$ must hold. Obviously, $\pi$ is a characteristic function of a fuzzy subset of $W$. Two measures on $W$ can be derived from $\pi$. They are called possibility and necessity measures and denoted by $\Pi$ and $N$ respectively. Formally, $\Pi, N: 2^{W} \rightarrow[0,1]$ are defined as $\Pi(S)=\sup _{w \in S} \pi(w)$ and $N(S)=1-\Pi(\bar{S})$, where $\bar{S}$ is the complement of $S$ with respect to $W$. Here, for convenience, we define $\sup \emptyset=0$ and $\inf \emptyset=1$.

Based on possibility theory, Dubois and Prade propose the possibilistic logic (PL)[4]. The wffs of PL are one of the forms $(\varphi N c)$ or $(\varphi \Pi c)$, where $\varphi$ is a classical logic formulas and $c \in(0,1]$. A model for PL is a possibility distribution $\pi: \Omega \rightarrow[0,1]$, where $\Omega$ is the set of all classical logic interpretations. For any classical wff $\varphi,|\varphi|=\{\omega \mid \omega \models \varphi\}$ is the truth set of $\varphi$. Then, the possibility and necessity measures of a classical logic wff can be defined via its truth set, i.e., $N(\varphi)=N(|\varphi|)$ and $\Pi(\varphi)=\Pi(|\varphi|)$. For a PL model $\pi$, define $\pi \models(\varphi N c)$ iff $N(\varphi) \geq c$ and $\pi=(\varphi \Pi c)$ iff $\Pi(\varphi) \geq c$.

To emphasize the qualitative aspect of possibilistic reasoning, a qualitative possibility logic (QPL) is proposed in [3]. While PL reasons about the possibility and necessity degrees of the wffs, QPL concerns mainly the relative comparison of possibility measures between two wffs. The syntax of QPL is an extension of propositional language with a binary connective " $\geq$ " and its wffs are defined by

$$
W F F:=p|\neg \varphi| \varphi \wedge \psi|\varphi \vee \psi| \varphi \supset \psi \mid \varphi \geq \psi
$$

The wff " $\varphi \geq \psi \wedge \neg(\psi \geq \varphi)$ " is abbreviated as " $\varphi>\psi$ ".

For the semantics, a QPL model is a triple $\mathcal{M}=(W, \pi, V)$, where $W$ is the set of possible worlds, $\pi: W \rightarrow[0,1]$ is a possibility distribution over $W$, and $V$ is a truth valuation which assigns to each propositional symbol a subset of $W$. The satisfaction relation of the modal formulas is as follows:

$$
\mathcal{M}, w \models \varphi \geq \psi \text { iff } \Pi(\varphi) \geq \Pi(\psi)
$$

where $\Pi(\varphi)$ and $\Pi(\psi)$ are defined via the truth sets of $\varphi$ and $\psi$ as in PL, though the truth sets in this case are subsets of possible worlds instead of classical interpretations.

\section{$4 \quad$ Hybrid Logic in Possibilistic Reasoning}

We have seen that the main motivation of hybrid logic is that modal logic lacks the capability of referring to the states. By using the nominals, hybrid logic thus extend the expressive power of modal logic. We would like to ask whether the same extension could be made for the possibilistic logic. The following would be the preliminary attempts to the answer of the question. 


\subsection{Hybrid possibilistic logic}

According to the syntax of PL, only possibility or necessity-qualified wffs are actually allowed. Thus PL is not an extension of propositional logic since propositional logic wffs are not those of PL. However, to hybridize PL, we must first add the propositional part to PL and then nominals can be seen as a special kind of propositional symbols as in basic hybrid logic. Thus, given a set of propositional symbols $\left\{p_{0}, p_{1}, \ldots\right\}$ and a set of nominals $\left\{a_{0}, a_{1}, \ldots\right\}$, the wffs of hybrid possibilistic logic (HPL) is defined as

$$
W F F:=a|p| \neg \varphi|\varphi \wedge \psi| \varphi \vee \psi|\varphi \supset \psi|[c] \varphi|\langle c\rangle \varphi| a: \varphi
$$

where $c$ is a numeral in $[0,1]$. Note that we adhere to a modal notation for the necessity and possibility-qualified wffs. The notation has been used in some graded modal formulation of possibilistic logic $[5,7]$.

An HPL model is a triple $\mathcal{M}=(W, \pi, V)$, where $W$ is the set of possible worlds, $\pi: W \rightarrow[0,1]$ is a possibility distribution over $W$, and $V$ is a truth valuation which assigns to each propositional symbol a subset of $W$ and each nominal a singleton subset of $W$. The conditions for the satisfaction of wffs are essentially same as those for HL except the modal operators which are defined as follows:

1. $\mathcal{M}, w=[c] \varphi$ iff $N(\varphi) \geq c$

2. $\mathcal{M}, w=\langle c\rangle \varphi$ iff $\Pi(\varphi) \geq c$

where $N(\varphi)$ and $\Pi(\varphi)$ is defined via the truth set of $\varphi$ and the possibility distribution $\pi$ as in the case of PL.

The semantics makes the truth values of the modal wffs in HPL independent of the worlds in which they are evaluated. In other words, $\mathcal{M}, w \models[c] \varphi$ for some world $w$ iff $\mathcal{M}, w \models[c] \varphi$ for all $w \in W$. However, we can have a more general semantics for the HPL if each world is associated with a (possibly) different possibility distribution. Indeed, this is particularly useful in similaritybased reasoning [5]. The hybrid logic for possibilistic reasoning based on the more general semantic setting has been studied in [6]. It is shown that hybrid logic may help the development of proof methods in the graded modal formulation of possibilistic reasoning. The logic is called hybrid graded modal logic(HGML) and its syntax is also a bit more general than that of HPL.

The wffs of HGML are defined as follows:

$$
W F F:=a|p| \neg \varphi|\varphi \wedge \psi| \varphi \vee \psi|\varphi \supset \psi|[c] \varphi\left|[c]^{+} \varphi\right|\langle c\rangle \varphi\left|\langle c\rangle^{+} \varphi\right| a: \varphi
$$

where $c$ is a numeral in $[0,1]$. Except satisfaction statements, the HGML language is just that of quantitative modal logic (QML) in [7]

The HGML language is interpreted on fuzzy hybrid models. Define an HGML model as a triple $\mathcal{M}=(W, R, V)$, where $W$ is a set of possible worlds (or states), $R: W \times W \rightarrow[0,1]$ is a fuzzy binary relation on $W$, and $V$ is a truth value assignment defined just as in the hybrid models. Given $R$, we can define 
a possibility distribution $\pi_{w}$ for each $w \in W$ such that $\pi_{w}(s)=R(w, s)$ for all $s \in W$.

Given a model $\mathcal{M}$, in addition to clauses for Boolean connectives and satisfaction statement in HL, those for modal wffs are as follows, where $\Pi_{w}$ and $N_{w}$ are the possibility and necessity measures corresponding to the possibility distribution $\pi_{w}$.

1. $\mathcal{M}, w \mid=\langle c\rangle \varphi \Leftrightarrow \Pi_{w}(\varphi) \geq c$,

2. $\mathcal{M}, w \mid=\langle c\rangle^{+} \varphi \Leftrightarrow \Pi_{w}(\varphi)>c$,

3. $\mathcal{M}, w=[c] \varphi \Leftrightarrow N_{w}(\varphi) \geq c$,

4. $\mathcal{M}, w=[c]^{+} \varphi \Leftrightarrow N_{w}(\varphi)>c$,

Note that $[c]^{+}$and $\langle c\rangle^{+}$correspond to the strict inequality of the uncertainty measures.

\subsection{Hybrid qualitative possibility logic}

It has been shown that the hybridization of possibilistic logic is helpful in the development of its proof methods[6]. However, for more practical applications, we can take the hybrid qualitative possibility logic(HQPL) as a tool for reasoning about multi-criteria decision making. Let us first define its syntax with respect to the set of propositional symbols, nominals, and a set of modality labels $\left\{\geq_{0}\right.$ $\left., \geq_{1}, \cdots\right\}$. Its wffs are

$$
W F F:=a|p| \neg \varphi|\varphi \wedge \psi| \varphi \vee \psi|\varphi \supset \psi| \varphi \geq_{i} \psi \mid a: \varphi
$$

For the semantics, an HQPL model is a triple $\mathcal{M}=\left(W,\left(\pi_{i}\right)_{i \geq 0}, V\right)$ where $W$ is the set of states, each $\pi_{i}$ is a possibility distribution over $W$, and $V$ is the truth valuation as in the HL models. In addition to the satisfaction clauses of HL, we have

$$
\mathcal{M}, w=\varphi \geq_{i} \psi \text { iff } \Pi_{i}(\varphi) \geq \Pi_{i}(\psi)
$$

where $\Pi_{i}$ is the possibility measure for $\pi_{i}$ and defined via the truth set of $\varphi$ and $\psi$.

In practical applications, each modality label can correspond to preference relation under a decision criterion, while the nominals are exactly the options available to the decision maker. There are in general two kinds of preference statements for the multi-criteria decision-making problems. The first is about the description of the general preference. This can be modelled by the QPL wff $\varphi \geq_{i} \psi$ which means that some options satisfying $\varphi$ are preferred than some ones satisfying $\psi$ according to the criterion $i$. The second regards the preference between specific options. This can only be modelled by wffs of the form $a \geq_{i} b$ which means that the option $a$ is preferred to $b$ according to the criterion $i$.

\subsection{Hybrid possibilistic description logic}

Some works on the application of fuzzy description or modal logics to information retrieval have been done previously $[9,8]$. However, following the tradition of DL, 
most approaches separate the terms and formulas. Here, we shows a hybrid logic approach where the objects to be retrieved and the queries are treated uniformly.

Let propositional symbols and nominals be given as above and $\left\{\alpha_{0}, \alpha_{1}, \cdots\right\}$ be a set of role names, then the syntax for the wffs of hybrid possibilistic description $\operatorname{logic}(\mathrm{HPDL})$ is as follows:

$$
\begin{aligned}
W F F:= & a|p| \neg \varphi|\varphi \wedge \psi| \varphi \vee \psi|\varphi \supset \psi| a: \varphi \mid \\
& {[c]_{i} \varphi\left|[c]_{i}^{+} \varphi\right|\langle c\rangle_{i} \varphi\left|\langle c\rangle_{i}^{+} \varphi\right|\left[\alpha_{i}\right] \varphi \mid\left\langle\alpha_{i}\right\rangle \varphi }
\end{aligned}
$$

where $c$ is still a numeral in $[0,1]$.

An HPDL model for the language is a 4-tuple $\left(W,\left(R_{i}\right)_{i \geq 0},\left(S_{i}\right)_{i \geq 0}, V\right)$, where $W$ and $V$ are defined as in the HL models, each $R_{i}$ is a (crisp) binary relation over $W$ and each $S_{i}$ is a fuzzy binary relation over $W$. In the application, it is especially assumed that each $S_{i}$ is in fact a similarity relation. A fuzzy relation $S: W \times W \rightarrow[0,1]$ is called a $\otimes$-similarity relation if it satisfies the following three conditions:

(i) reflexivity: $S(w, w)=1$ for all $w \in W$

(ii) symmetry: $S(w, u)=S(u, w)$ for all $w, u \in W$

(iii) $\otimes$-transitivity: $S(w, u) \geq \sup _{x \in W} S(w, x) \otimes S(x, u)$ for all $w, u \in W$

where $\otimes$ is a t-norm ${ }^{1}$. The semantics for modalities based on role names is the same as that for ML and for the similarity-based modalities, we adopt the semantics of HGML, so we do not have to repeat it again.

In the application, the model can have an information retrieval interpretation, where $W$ denotes the set of objects (documents, images, movies, etc.) to be retrieved and each $S_{i}$ is an endowed similarity relations which is associated with some aspect such as style, color, etc. As for the truth valuation function $V$ and the relations $R_{i}$ 's, they decide the extensions of each wff just like the interpretation function of DL, so each wff in HPDL also corresponds to a concept term in DL. Since nominals are just a special kinds of wffs and each nominal refers to a retrievable object, the objects in the model are also denoted by wffs in the same way as queries. Let us look at an example adapted from [8] to illustrate the use of the logic.

Example 1 (Exemplar-based retrieval) In some cases, in particular, for the retrieval of multimedia information, we may be given an exemplar or standard document and try to find documents very similar to the exemplar but satisfying some additional properties. In this case, we can formulate the query as $\langle c\rangle a \wedge \varphi$, where $a$ is the name for the exemplar and $\varphi$ denotes the additional desired properties. According to the semantics, $b:\langle c\rangle a$ will be satisfied by an HPDL model $\left(W,\left(R_{i}\right)_{i \geq 0}, S, V\right)$ iff $S(V(a), V(b)) \geq c$. Thus, a document referred by $b$ will meet the query if it can satisfy the properties denoted by $\varphi$ and is similar to the exemplar at least with degree $c$.

\footnotetext{
${ }^{1}$ A binary operation $\otimes:[0,1]^{2} \rightarrow[0,1]$ is a t-norm iff it is associative, commutative, and increasing in both places, and $1 \otimes x=x$ and $0 \otimes x=0$ for all $x \in[0,1]$.
} 
It has been shown that the hybridization of DL in fact makes it possible to accommodate more expressive powers than $\mathcal{A L C}[2]$. In particular, it can express number restriction and collection of individuals. For example, $b:($ book $\wedge$ 〈author $a_{1} \wedge\langle$ author $\rangle a_{2} \wedge \neg a_{1}: a_{2}$ ) means that $b$ is a book with at lease two authors.

\section{Conclusion}

We have presented some preliminary proposals on the hybridization of possibilistic logic in this paper. We study some variants of hybrid possibilistic logic and show that some application domains such as multi-criteria decision making and information retrieval indeed benefit from the hybridization. Further works on the elaboration of the proposed logical systems are expected.

\section{References}

1. P. Blackburn. "Representation, reasoning, and relational structures: a hybrid logic manifesto". Logic Journal of IGPL, 8(3):339-365, 2000.

2. P. Blackburn and M. Tzakova. "Hybridizing concept languages". Annals of Mathematics and Artificial INtelligence, 24:23-49, 1999.

3. L. Farinas del Cerro and A. Herzig. "A modal analysis of possibility theory". In R. Kruse and P. Siegel, editors, Proceedings of the 1st ECSQAU, LNAI 548, pages 58-62. Springer-Verlag, 1991.

4. D. Dubois, J. Lang, and H. Prade. "Possibilistic logic". In D.M. Gabbay, C.J. Hogger, and J.A. Robinson, editors, Handbook of Logic in Artificial Intelligence and Logic Programming, Vol 3 : Nonmonotonic Reasoning and Uncertain Reasoning, pages 439-513. Clarendon Press - Oxford, 1994.

5. F. Esteva, P. Garcia, L. Godo, and R. Rodriguez. "A modal account of similaritybased reasoning". International Journal of Approximate Reasoning, pages 235-260, 1997.

6. C.J. Liau. "Hybrid logic for possibilistic reasoning". In Proc. of the Joint 9th IFSA World Congress and 20th NAFIPS International Conference, pages 15231528, 2001.

7. C.J. Liau and I.P. Lin. "Quantitative modal logic and possibilistic reasoning". In B. Neumann, editor, Proceedings of the 10th ECAI, pages 43-47. John Wiley \& Sons. Ltd, 1992.

8. C.J. Liau and Y.Y. Yao. "Information retrieval by possibilistic reasoning". In H.C. Mayr, J. Lazansky, G. Quirchmayr, and P. Vogel, editors, Proc. of the 12th International Conference on Database and Expert Systems Applications (DEXA2001),, LNCS 2113, pages 52-61. Springer-Verlag, 2001.

9. C. Meghini, F. Sebastiani, and U. Straccia. "A model of multimedia information retrieval". JACM, 48(5):909-970, 2001.

10. M. Schmidt-Schauß and G. Smolka. "Attributive concept descriptions with complements". Artificial Intelligence, 48(1):1-26, 1991.

11. L.A. Zadeh. "Fuzzy sets as a basis for a theory of possibility". Fuzzy Sets and Systems, 1(1):3-28, 1978. 